\title{
TERF1 downregulation promotes the migration and invasion of the PC3 prostate cancer cell line as a target of miR-155
}

\author{
WEI CHEN ${ }^{1,2 *}$, LI-NA HE ${ }^{3 *}$, YONG LIANG ${ }^{2}$, XIANG ZENG $^{2}$, CUI-PING WU ${ }^{1}$, \\ MING-QIANG SU ${ }^{2}$, YANG $\mathrm{CHENG}^{2}$ and JIAN-HUI LIU ${ }^{2}$ \\ Departments of ${ }^{1}$ Science and Education and ${ }^{2}$ Urology, Zigong Fourth People's Hospital; \\ ${ }^{3}$ Department of Reproductive Medicine, Zigong Maternity and Child Healthcare Hospital, \\ Zigong, Sichuan 643000, P.R. China
}

Received March 28, 2020; Accepted September 28, 2020

DOI: $10.3892 / \mathrm{mmr} .2020 .11623$

\begin{abstract}
Telomeric repeat binding factor 1 (TERF1) has been identified as a tumor suppressor gene in numerous types of human cancer. However, the expression of TERF1 and its mechanism in prostate cancer $(\mathrm{PCa})$ remains unclear. The present study aimed to explore the expression and functions of TERF1 in PCa. The UALCAN database was used to analyze the differential expression of TERF1 between normal prostate tissue and primary PCa tissue. Cell apoptosis was analyzed by Annexin V/propidium iodide staining, and wound healing and Transwell assays were used to detect the cell migration and invasion abilities, respectively. The cell viability was analyzed using an MTT assay. Reverse transcription-quantitative PCR and western blotting were used to analyze the mRNA and protein expression levels, respectively, of epithelial-mesenchymal transition (EMT) markers following TERF1 knockdown in the PC3 cell line. A dual luciferase reporter assay was used to verify the association between TERF1 and microRNA (miR)-155 predicted by bioinformatics analysis. Rescue experiments were performed to determine the role of the miR-155/TERF1 axis in regulating the cellular behaviors of PCa. The results demonstrated that the expression levels of TERF1 in the primary prostate tumors were significantly downregulated compared with in prostate normal tissue. TERF1 silencing was discovered to significantly promote cell viability, migration and invasion, while suppressing cell apoptosis. The impact of TERF1 on PC3 cells was suggested to occur through the EMT pathway. TERF1 was confirmed to be the direct target of miR-155. The overexpression of
\end{abstract}

Correspondence to: Dr Jian-Hui Liu, Department of Urology, Zigong Fourth People's Hospital, 9/F Inpatient Building, 19 Tanmulin Street, Ziliujing, Zigong, Sichuan 643000, P.R. China E-mail: urologyly@163.com

${ }^{*}$ Contributed equally

Key words: telomeric repeat binding factor 1, prostate cancer, microRNA-155, epithelial-mesenchymal transition
miR-155 promoted the viability, migration and invasion, while suppressing the apoptosis of the PC3 cell line, while the knockdown of miR-155 in PC3 cells achieved the opposite trends. In addition, TERF1 overexpression reversed the promotive effects of upregulated miR-155 expression levels on the migration and apoptosis of PC 3 cells. On the contrary, the knockdown of TERF1 reversed the migration and apoptosis abilities of the downregulated miR-155 expression levels on the cellular behaviors of PC3 cells. In conclusion, TERF1, as a direct target of miR-155, was discovered to be significantly downregulated in $\mathrm{PCa}$, which was suggested to promote the migration and invasion of PCa via the EMT pathway.

\section{Introduction}

Accounting for $6.6 \%$ (307,471 cases) of all cancer mortality in men, prostate cancer $(\mathrm{PCa})$ is the most common solid malignancy in men worldwide and the second highest cause of cancer-associated mortality in western countries $(1,2)$. The early diagnosis of PCa may improve the overall survival and progression-free survival. Currently, a serum prostate-specific antigen (PSA) test and digital rectal examination are the main screening strategies for PCa in the clinic (3). However, the PSA level may be influenced by confounding factors, such as urinary tract infection, inflammation or transurethral operation (4). Androgen deprivation therapy is the first-line therapeutic method for reducing circulating androgen levels and tumor growth; however, usually after 2-3 years, the patients develop hormone-refractory $\mathrm{PCa}$ and progresses to castration-resistant $\mathrm{PCa}$ (CRPC) (1,5). Therefore, determining the potential mechanisms of PCa progression and identifying novel biomarkers is important for the diagnosis and treatment of PCa.

Telomeric repeat binding factor 1 (TERF1) is an important telomeric binding protein and is vital for the protection and maintenance of telomere DNA in mammalian cells (6). Several studies have reported the downregulation of TERF1 in numerous types of cancer; therefore, it may be a potential marker for cancer diagnosis $(7,8)$. Nevertheless, the mechanism of TERF1 in PCa remains unclear. Thus, investigations into the function of TERF1 may provide novel insights into the molecular mechanisms of PCa. MicroRNAs (miRNAs/miRs) are a set of small non-coding RNA molecules of 18-25 nucleotides in 
length that negatively mediate gene expression through binding to the 3'-untranslated region (3'-UTR) of target mRNAs $(9,10)$. Multiple studies have demonstrated that miRNAs are involved in the regulation of various biological processes such as cancer invasion or migration by targeting the majority of protein-coding genes $(11,12)$. Accumulating data has suggested that the abnormal expression of miRNAs was implicated in the progression of $\mathrm{PCa}$ through numerous signaling pathways, including Notch and HIF-1 $\alpha$ pathways (13-16). Hence, it is necessary to determine the functions of miRNAs in $\mathrm{PCa}$, which may reveal a novel mechanism of PCa progression.

Bioinformatics analysis in the present study using online tool miRWalk and data from the The Cancer Genome Atlas database indicated that TERF1 may serve as a tumor suppressor in PCa. Moreover, miR-155 was discovered to directly bind to the 3'-UTR of TERF1 (17). The present study demonstrated an essential role of the miR-155/TERF1 axis in the progression of PCa.

\section{Materials and methods}

Cell culture and transfection. The human prostate cancer cell line, PC3, was purchased from the American Type Culture Collection (ATCC). PC3 cells were cultured in RPMI-1640 medium (Thermo Fisher Scientific, Inc.) supplemented with 10\% FBS, (Gibco; Thermo Fisher Scientific, Inc) , and maintained at $37^{\circ} \mathrm{C}$ in a humidified atmosphere of $5 \% \mathrm{CO}_{2}$.

PC 3 cells $\left(2 \times 10^{5}\right.$ cells/well) were cultured until $60 \%$ confluence and transiently transfected with $50 \mathrm{nmol} / \mathrm{l}$ agomiR-155 (Guangzhou RiboBio Co., Ltd.) or agomiR-NC (Guangzhou RiboBio Co., Ltd.) to overexpress miR-155, antagomiR-155 (Guangzhou RiboBio Co., Ltd.) or antagomiR-NC (Guangzhou RiboBio Co., Ltd.) to downregulate miR-155 expression, TERF1-siRNA (5'-GGAAGUUACUUAAGAUAAUCU-3'; Guangzhou RiboBio Co., Ltd.) or siRNA NC (5'-AATTCT CCGAACGTGTCACGT-3'; Guangzhou RiboBio Co., Ltd.) to downregulate TERF1 expression levels, or pcDNA3.1-TERF1 (Invitrogen; Thermo Fisher Scientific, Inc) overexpression plasmid to overexpress TERF1 using Lipofectamine ${ }^{\circledR} 2000$ reagent (Invitrogen; Thermo Fisher Scientific, Inc.). Empty vector was used as the negative control. Transfected cells were cultured at $37^{\circ} \mathrm{C}$ for $6 \mathrm{~h}$ prior to the replacement of complete medium. Following 24-72 h, the transfected cells were harvested for in vitro experiments depending on the different assay. The transfection efficiencies were analyzed using reverse transcription-quantitative PCR (RT-qPCR).

The cells were divided into the following groups: i) Mock control group (without transfection); ii) TERF1-small interfering RNA (siRNA) negative control (NC) group (transfected with NC siRNA); iii) TERF1-siRNA group (transfected with TERF1 siRNA); iv) agomiR-155 NC group (transfected with agomiR-155 NC); v) agomiR-155 group (transfected with agomiR-155); vi) antagomiR-155 NC group (transfected with antagomiR-155 NC); vii) antagomiR-155 group (transfected with antagomiR-155); viii) agomiR-155 + TERF1 group (transfected with agomiR-155 and pcDNA3.1-TERF1 plasmid); and ix) antagomiR-155 + TERF1-siRNA group (transfected with antagomiR-155 and TERF1-siRNA).

Flow cytometric analysis of apoptosis. To determine the levels of apoptosis, PC3 cells (after transfection for $48 \mathrm{~h}$ ) were washed with PBS and centrifuged at $300 \mathrm{x}$ g at $4^{\circ} \mathrm{C}$ for $4 \mathrm{~min}$ 3 days post-transfection. The cells were resuspending in $100 \mu \mathrm{l}$ binding buffer and incubated with $5 \mu \mathrm{l}$ Annexin V-FITC and $10 \mu \mathrm{l}$ propidium iodide (PI) in the dark at room temperature for $30 \mathrm{~min}$. Following the incubation, $400 \mu \mathrm{l}$ binding buffer was added and the apoptotic cells were analyzed by flow cytometry (FACSCanto II; BD Biosciences) and software FlowJo (version 7.6.3; FlowJo LLC). The percentage of early apoptotic cells were calculated to evaluate the difference between groups.

Wound healing assay. PC3 cells (after transfection for $24 \mathrm{~h}$ ) were trypsinized at $37^{\circ} \mathrm{C}$ until the cell layer was suspended and then centrifuged at $300 \mathrm{xg}$ at room temperature for $5 \mathrm{~min}$. The cells were resuspended in RPMI-1640 medium and seeded in a 6 -well cell culture plate at a density of $4 \times 10^{5}$ cells/well. Upon the cells reaching $90-100 \%$ confluence, vertical linear scratches were made in the cell monolayer using a sterile $200-\mu 1$ pipette tip. The suspended cells were removed by washing with PBS and the adhered cells were subsequently incubated with serum-free RPMI-1640 medium. Images of the scratches were photographed under an inverted light microscope with 10x magnification at 0 and $48 \mathrm{~h}$. The percentage of the healed wound area was measured by ImageJ (version 5.0; Bio-Rad Laboratories, Inc.).

Matrigel invasion assay. To analyze cell invasion, Transwell chambers were precoated with $100 \mu \mathrm{l}$ Matrigel diluted to $50 \mu \mathrm{g} / \mathrm{ml}$ with DMEM at $37^{\circ} \mathrm{C}$ for $2 \mathrm{~h}$. PC3 cells (after transfection for $24 \mathrm{~h}$ ) were harvested by centrifuging at $300 \mathrm{x} \mathrm{g}$ at room temperature for $5 \mathrm{~min}$ and $2 \times 10^{4}$ cells were seeded into the upper chamber in a serum-free RPMI-1640 medium. RPMI-1640 medium supplemented with 10\% FBS was added to the lower chambers. Following incubation for $48 \mathrm{~h}$ at $37^{\circ} \mathrm{C}$, non-invasive cells on the upper surface of the filter were removed with a cotton swab. The remaining invasive cells were fixed by submerging in $10 \%$ formalin for $10 \mathrm{~min}$ at room temparature, then washed with PBS once. Fixed cells were stained with $0.5 \%$ hematoxylin for $30 \mathrm{~min}$ at room temperature and counted under a light microscope (magnification, x200) in three randomly selected fields of view.

MTT assay. Cells in the exponential phase were harvested by centrifuging at $300 \mathrm{x} \mathrm{g}$ at room temperature for $5 \mathrm{~min}$ and resuspended in RPMI-1640 medium. The cells were plated into a 96-well cell culture plate at a density of $1 \times 10^{3}$ cells/well and cultured at $37^{\circ} \mathrm{C}$ and $5 \% \mathrm{CO}_{2}$ for 1-7 days. The cells were then incubated for $4 \mathrm{~h}$ with $5 \%$ MTT solution (20 $\mu \mathrm{l} /$ well) in the dark at $37^{\circ} \mathrm{C}$. Plates were then treated with $100 \mu \mathrm{l}$ $\mathrm{DMSO} /$ well in the dark to dissolve the purple formazan crystals. Subsequently, a microplate reader (SAF-680T; Jiangsu Baju Pharmaceutical Co. Ltd.) was used to measure the optical density (OD) at a wavelength of $490 \mathrm{~nm}$. Growth curves of the cells were plotted with the OD value on the $y$-axis and time on the $\mathrm{x}$-axis. The assays were conducted three times independently.

Dual luciferase reporter assay. The putative wild-type (WT) miR-155 complementary binding site in the 3'-UTR of TERF1 was amplified by PCR. A mutant (Mut) construct in the 
Table I. Primer sequences for reverse transcription-quantitative PCR.

\begin{tabular}{ll} 
Gene & \multicolumn{1}{c}{ Primer sequence $\left(5^{\prime} \rightarrow 3^{\prime}\right)$} \\
\hline GAPDH & F: ATGGCCTTCCGTGTTCCTAC \\
Telomeric repeat binding factor 1 & F: CTTTACAAAGTTGTCGTTGA \\
& R: TTGCCGCTCTAACACAGGCTGG \\
E-cadherin & F: TGGCTTCCCTCTTTCATCATA \\
Vimentin & R: GTTCCGCTCTGTCTTTGG \\
N-cadherin & F: TGATTAAGACGGTTGAAACTAG \\
U6 & R: AGAAAGGCACTTGAAAGCT \\
& F: TTTGATGGAGGTCTCCTAACACC \\
MicroRNA-155 & R: ACGTTTAACACGTTGGAAATGTG \\
& F: CTCGCTTCGGCAGCAC \\
& R: AACGCTTCACGAATTTGCGT \\
& F: ACACTCCAGCTGGGTTAATGCTAATCGTGA
\end{tabular}

F, forward; R, reverse.

miR-155 binding site of the TERF1 3'-UTR region was also generated using a Quick-Change Site-Directed Mutagenesis kit (Agilent Technologies, Inc.) and named Mut-TERF1 3'-UTR. The 3'-UTR of TERF1 or its Mut sequence were cloned into the pmiRRB-REPORT vector (Guangzhou RiboBio Co., Ltd.). Then, WT-TERF1 3'-UTR or Mut-TERF1 3'-UTR were co-transfected using Lipofectamine ${ }^{\circledR} 2000$ reagent (Invitrogen; Thermo Fisher Scientific, Inc.) into 293 T cells (ATCC; $1 \times 10^{5}$ cells/well) with agomiR-155 or antagomiR-155 in 48-well plates. The relative luciferase activity was measured using a Dual-Luciferase Reporter assay system (Promega Corporation) following transfection for $48 \mathrm{~h}$ at $37^{\circ} \mathrm{C}$ following $48 \mathrm{~h}$ of transfection. All data in dual luciferase reporter assay were normalized to Renilla luciferase activity. Each assay was independently repeated three times.

$R T$-qPCR. Total RNA was extracted from PC3 cells using TRIzol ${ }^{\circledR}$ reagent (Invitrogen; Thermo Fisher Scientific, Inc.) Total RNA $(2 \mu \mathrm{g})$ was reverse transcribed into cDNA using First Strand cDNA Synthesis kit (Invitrogen; Thermo Fisher Scientific, Inc.) at $37^{\circ} \mathrm{C}$ for $15 \mathrm{~min}$, and cDNA was incubated at $85^{\circ} \mathrm{C}$ for $5 \mathrm{sec}$ to inactivate the reverse transcriptase. qPCR was subsequently performed using a SYBR Premix Ex Taq II kit (Takara Bio, Inc.). The following thermocycling conditions were used for the qPCR: Initial denaturation at $94^{\circ} \mathrm{C}$ for $5 \mathrm{~min}$; followed by 40 cycles at $94^{\circ} \mathrm{C}$ for $45 \mathrm{sec}, 55^{\circ} \mathrm{C}$ for $30 \mathrm{sec}$ and $72^{\circ} \mathrm{C}$ for $45 \mathrm{sec}$. The primer sequences used for the qPCR are listed in Table I. Expression levels were quantified using the $2^{-\Delta \Delta \mathrm{Cq}}$ method (18). mRNA expression levels were normalized to the internal loading control, GAPDH, while miRNA expression levels were normalized to the internal loading control, U6. All samples were run in triplicate.

Western blotting. Cells were cultured in complete RPMI-1640 medium at $37^{\circ} \mathrm{C}$ for 3 days and total protein was extracted using $120 \mu \mathrm{l}$ ice-cold RIPA buffer (Sangon Biotech Co., Ltd., Shanghai, China). Protein concentration was quantified using a Bradford protein assay kit (Beyotime Institute of Biotechnology) and $50 \mu \mathrm{g}$ protein/lane was separated via $10 \%$ SDS-PAGE. The separated proteins were subsequently transferred onto PVDF membranes (Invitrogen, Thermo Fisher Scientific, Inc.) for $2 \mathrm{~h}$ and then blocked with 5\% BSA (Gibco, Thermo Fisher Scientific, Inc.)at room temperature for $1 \mathrm{~h}$. The membranes were then incubated with the following primary antibodies at $4^{\circ} \mathrm{C}$ overnight: Anti-TERF1 ( ab10579; Abcam; 1:1,000), anti-E-cadherin (cat. no. ab40772, Abcam; 1:500), anti-N-cadherin (cat. no. ab98952, Abcam; 1:500), anti-Vimentin (cat. no. ab217673, Abcam; 1:1,000) and anti-GAPDH (cat. no. ab181603, Abcam; 1:1,000). Following the primary antibody incubation, the membranes were washed three times with $0.1 \%$ PBS-Tween-20 (PBST) and then incubated with HRP-conjugated goat anti-rabbit or -mouse IgG secondary antibody for $1 \mathrm{~h}$ at room temperature. Protein bands were visualized using an ECL reagent (Invitrogen; Thermo Fisher Scientific, Inc.) and a Fusion FX5 image analysis system (Vilber Lourmat) after the final wash with PBST three times was completed. The densitometric analysis of protein was measured using ImageJ (version 5.0; Bio-Rad Laboratories, Inc.).

Bioinformatics prediction. The online bioinformatics analysis website miRWalk (mirwalk.umm.uni-heidelberg.de) (19) was used to predict the complementary binding sites for miR-155 in the 3'-UTR of target genes. The UALCAN database (http://ualcan.path.uab.edu/index.html) (20) was used to determine the expression levels of TERF1 between normal prostate tissue and primary PCa tissue. The assigned Gleason score in the UALCAN database was referenced from The 2014 International Society of Urological Pathology Consensus Conference on Gleason Grading of Prostatic Carcinoma (21). 

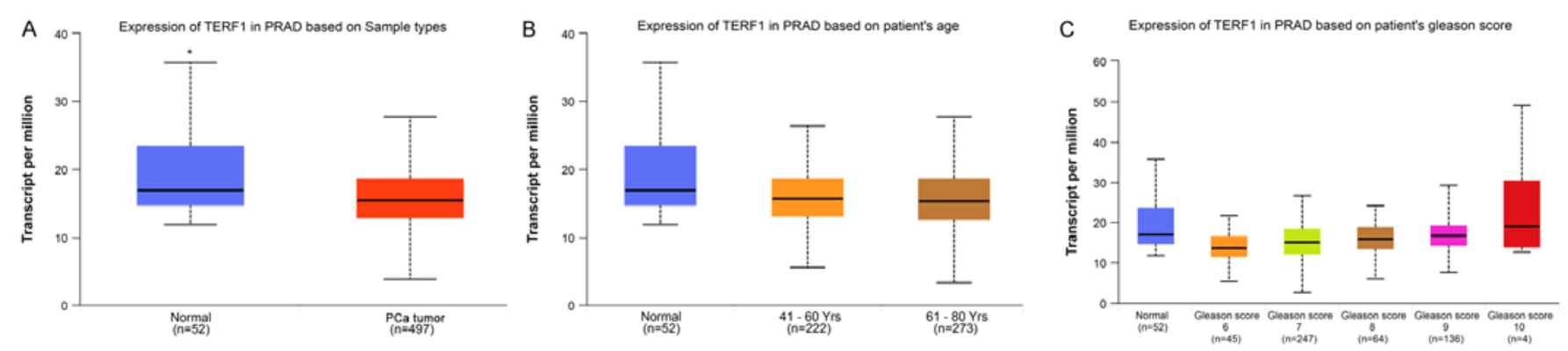

Figure 1. Expression levels of TERF1 in prostate tissues according to the UALCAN database. (A) Expression levels of TERF1 in PCa tumor and normal prostate tissues. "P<0.05 vs. PCa tumor. Expression levels of TERF1 in patients with PCa according to (B) age and (C) the patient's Gleason score. PRAD, prostate adenocarcinoma; TERF1, telomeric repeat binding factor 1; $\mathrm{PCa}$, prostate cancer.

Statistical analysis. All data from at $\geq 3$ independent experiments are presented as the mean \pm SEM. A two-tailed unpaired Student's t-test was used to compare the statistical differences between two groups, while a one-way ANOVA and a Bonferroni's post hoc test were used to compare the statistical differences among multiple groups. Analysis was performed using SPSS (version 20.0; IBM Corp.). $\mathrm{P}<0.05$ was considered to indicate a statistically significant difference.

\section{Results}

TERF1 expression levels are downregulated in PCa. To investigate the role of TERF1 in human PCa, the UALCAN database was used to analyze the expression levels of TERF1in 52 normal prostate tissue samples and 497 primary PCa tissue samples. The results revealed that the expression levels of TERF1 were significantly downregulated in primary PCa compared with normal prostate tissue samples (Fig. 1A). Nevertheless, both the patient's age and Gleason score exhibited no significant association with TERF1 (Fig. 1B and C).

Downregulation of TERF1 expression levels promotes the progression of $\mathrm{PCa}$ in vitro. To investigate the functions of TERF1 in PCa in vitro, TERF1-siRNA was transfected into PC 3 cells. The transfection efficiency of the knockdown of TERF1 using siRNA in PC3 cells was initially confirmed by RT-qPCR (Fig. 2E). Flow cytometric analysis of apoptosis revealed that the knockdown of TERF1 significantly inhibited the apoptosis of PC3 cells compared with the mock control and TERF1-siRNA NC groups (Fig. 2A). The effect of TERF1 knockdown on cell invasion and migration was analyzed using Transwell and wound healing assays, respectively; the results indicated that the number of invasive and migratory cells were significantly increased in the TERF1-siRNA group compared with the mock control and TERF1-siRNA NC groups (Fig. 2B and C). Furthermore, an MTT assay demonstrated that the knockdown of TERF1 expression significantly increased the cell viability of PC 3 cells compared with the TERF1-siRNA NC and mock control groups at days 1-7 (Fig. 2D).

Downregulation of TERF1 expression levels promotes epithelial-mesenchymal transition (EMT) in PCa cells. Due to the suppressive role of TERF1 on the progression of cultured PC 3 cells, the present study subsequently investigated whether the EMT pathway participated in this process. RT-qPCR analysis revealed that the transfection of PC 3 cells with TERF1-siRNA resulted in significantly upregulated expression levels of $\mathrm{N}$-cadherin and vimentin compared with the TERF1-siRNA NC and mock control groups (Fig. 3A). However, the expression levels of E-cadherin were significantly downregulated following the transfection with TERF1-siRNA compared with the TERF1-siRNA NC and mock control groups (Fig. 3A). Western blotting analysis revealed an identical trend at the protein level (Fig. 3B). In summary, these findings suggested that the knockdown of TERF1 expression levels may promote EMT in $\mathrm{PCa}$, and therefore may serve a role in PCa metastasis (Fig. 3C).

TERF1 is a direct target of miR-155. To further investigate the mechanism of TERF1 in promoting PCa progression, bioinformatics analysis using miRWalk was used to predict the potential miRNAs that regulate TERF1 (Fig. 4A). miR-155 was selected as a candidate, which has been reported as an oncogenic-associated molecule in other types of cancer (17). Subsequently, a dual luciferase reporter assay was used to verify whether miR-155 could directly bind to the TERF1 3'-UTR. Compared with mock control group, the results revealed that the relative luciferase activity of the WT-TERF1 3'-UTR in PC3 cells was significantly repressed following the co-transfection with the agomiR-155 (Fig. 4B). However, there was no statistical difference observed in the relative luciferase activity when PC3 cells were co-transfected with the WT-TERF1 3'-UTR and antagomiR-155. When compared with mock control group, the dual luciferase reporter assay also indicated that the upregulation of miR-155 did not affect the relative luciferase activity of the Mut-TERF1 3'-UTR in PC3 cells (Fig. 4B). In addition, a negative association was identified between the expression levels of miR-155 and TERF1. The expression levels of TERF1 were significantly upregulated following the knockdown of miR-155 compared with the mock control group, while the expression levels of TERF1 were significantly downregulated following the overexpression of miR-155 compared with the mock control group (Fig. 4C and D). These results indicated that miR-155 may directly target the TERF1 3'-UTR to participate in the progression of $\mathrm{PCa}$.

miR-155 promotes the migration and invasion of $P C a$ by targeting TERF1. To confirm the association between miR-155 and TERF1 and their role in PCa metastasis, the 
A

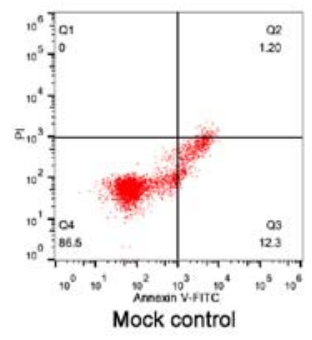

B

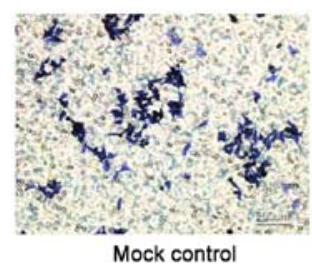

C
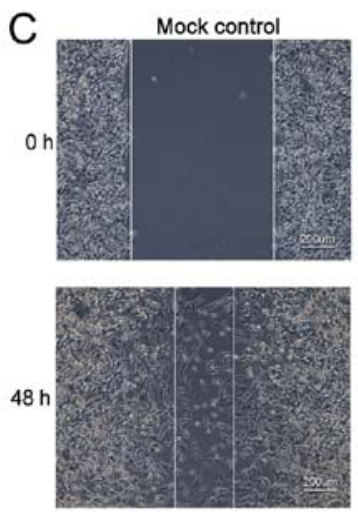
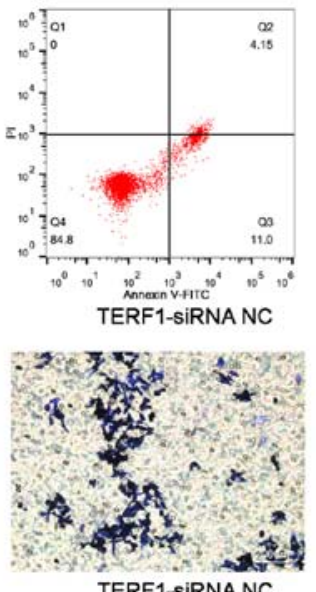

TERF1-siRNA NC
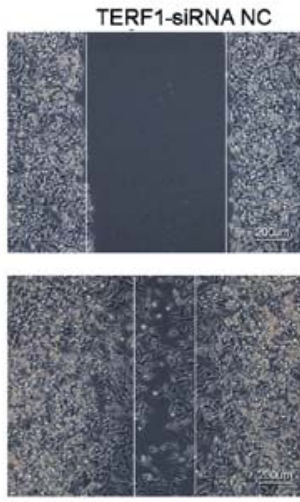
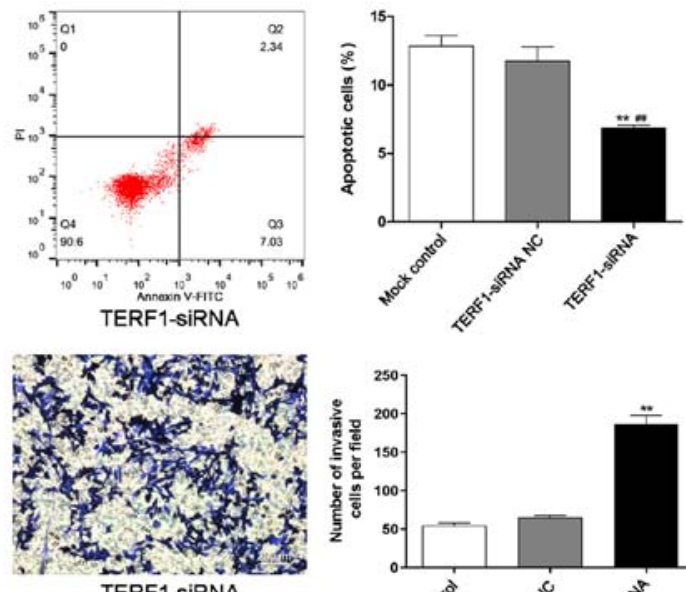

TERF1-siRNA
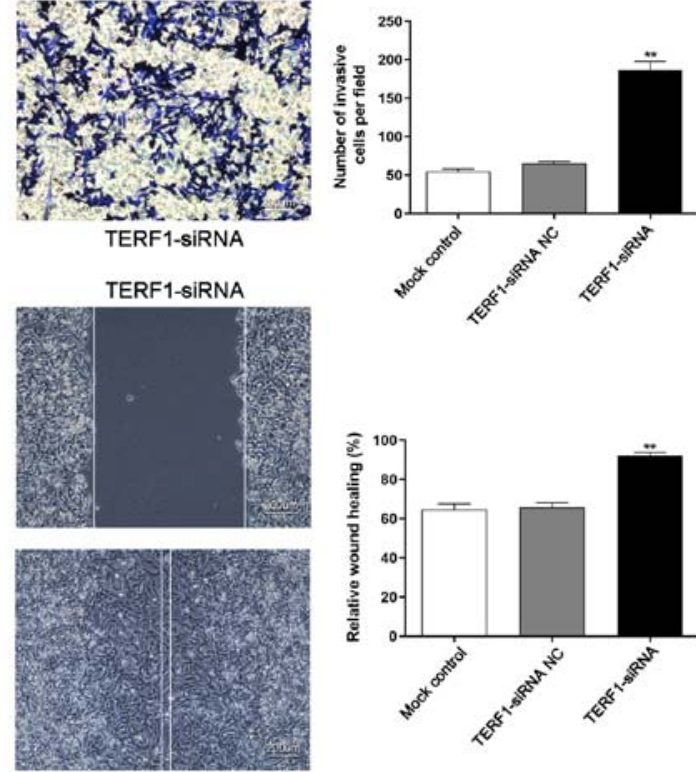

$\mathrm{E}$

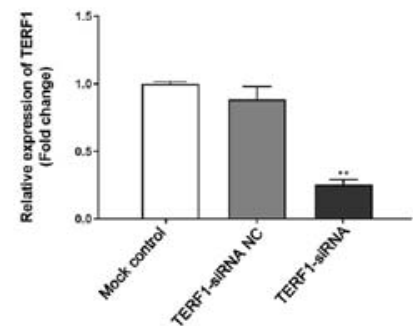

Figure 2. Knockdown of TERF1 promotes the viability, invasion and migration of the PC3 cell line, while inhibiting apoptosis. (A) Levels of apoptosis in PC3 cells were analyzed using flow cytometry. (B) Invasive ability of PC3 cells was analyzed using a Transwell assay. Scale bar, 200- $\mu$ m. (C) Migratory ability of PC3 cells was analyzed using a wound healing assay. Scale bar, 200- $\mu \mathrm{m}$. (D) Viability of PC3 cells was analyzed using an MTT assay. (E) Transfection efficiency of TERF1 knockdown in PC3 cells was analyzed using reverse transcription-quantitative $\mathrm{PCR}$. ${ }^{* *} \mathrm{P}<0.01$ vs. mock control; ${ }^{\# \#} \mathrm{P}<0.01$ vs. TERF1-siRNA NC. TERF1, telomeric repeat binding factor 1; PI, propidium iodide; siRNA, small interfering RNA; NC, negative control; OD, optical density.

expression levels of miR-155 in PC3 cells were overexpressed and knocked down by the transfection with an agomiR-155 or antagomiR-155, respectively. Furthermore, pcDNA3.1-TERF1 plasmids and TERF1-siRNA oligonucleotides were used to regulate the expression levels of TERF1 in rescue experiments. Flow cytometric analysis of apoptosis revealed that the overexpression of miR-155 significantly inhibited the apoptosis of PC3 cells compared with the mock control and agomiR-155 NC groups (Fig. 5A). By contrast, the cells transfected with antagomiR-155 had significantly increased levels of cell apoptosis compared with the mock control and anatgomiR-155 NC groups (Fig. 6A). The transfection efficiencies were confirmed by RT-qPCR (Figs. 5E and 6E).

To investigate the effects of miR-155 on PCa invasion and migration, Transwell and wound healing assays, respectively, were performed; the results revealed that the upregulation of miR-155 significantly increased the number of invasive and migratory cells compared with the mock control and agomiR-155 NC groups (Fig. 5B and C). The data from the MTT assay illustrated that the overexpression of miR-155 could significantly promote the viability of PC3 cells compared with the mock control group at days 3-7 (Fig. 5D). Notably, TERF1 overexpression could reverse the promotive effects of upregulated miR-155 expression on the invasion, migration and viability of PC3 cells (Fig. 5B-D). In addition, the overexpression of TERF1 could reverse the inhibitory effects of the agomiR-155 on apoptosis (Fig. 5A). On the contrary, the knockdown of TERF1 expression reversed the inhibitory effects of the antagomiR-155 on the cellular behaviors of PC3 cells (invasion, migration and viability), while reversing the promoting effects of the antagomiR-155 on apoptosis (Fig. 6A-D). Taken together, the present data indicated that miR-155 may promote the progression of $\mathrm{PCa}$ by directly binding to TERF1. 
A

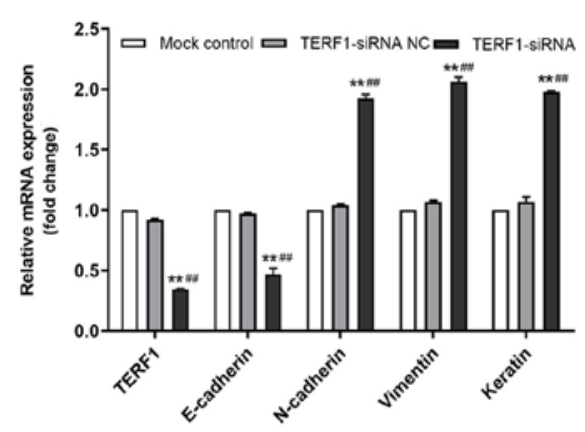

B

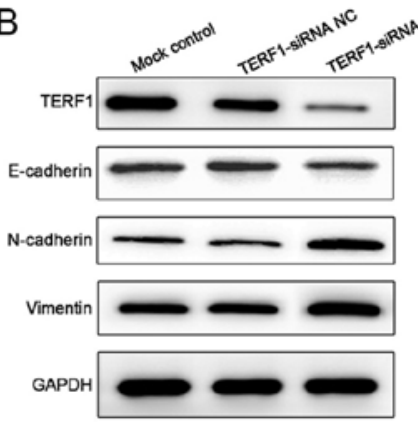

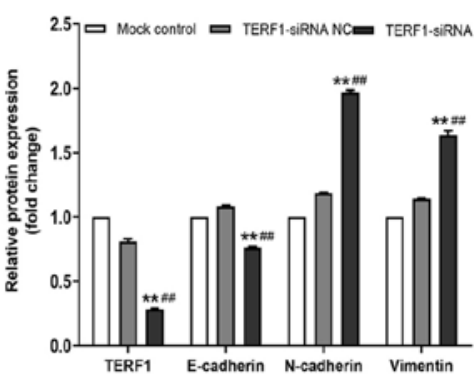

C PCa

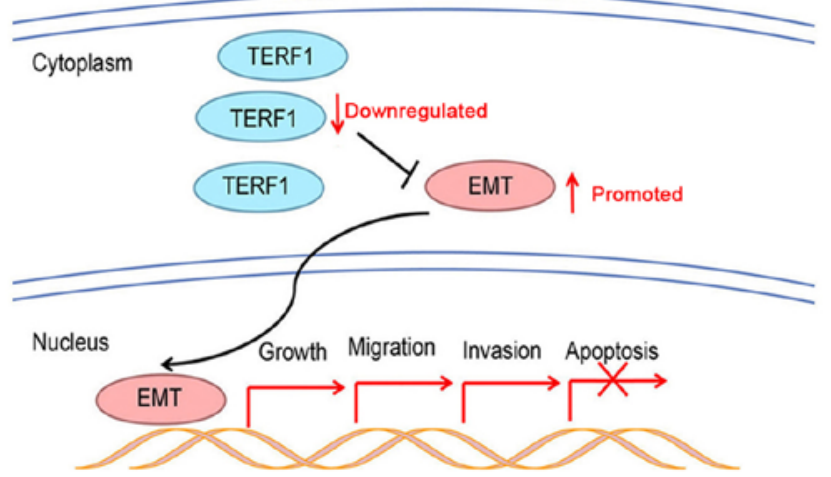

Figure 3. TERF1 promotes EMT in PC3 cells. (A) Reverse transcription-quantitative PCR analysis of TERF1, E-cadherin, N-cadherin and vimentin expression levels in PC3 cells following the knockdown of TERF1. (B) Western blotting was used to analyze the protein expression levels of TERF1, E-cadherin, $\mathrm{N}$-cadherin and vimentin in PC3 cells following the knockdown of TERF1. (C) Schematic diagram of the role of TERF1 in the progression of PCa via the EMT pathway. ${ }^{* *} \mathrm{P}<0.01$ vs. mock control; ${ }^{\# \#} \mathrm{P}<0.01$ vs. TERF1-siRNA NC. TERF1, telomeric repeat binding factor 1; PCa, prostate cancer; EMT, epithelial-mesenchymal transition; siRNA, small interfering RNA; NC, negative control.

A

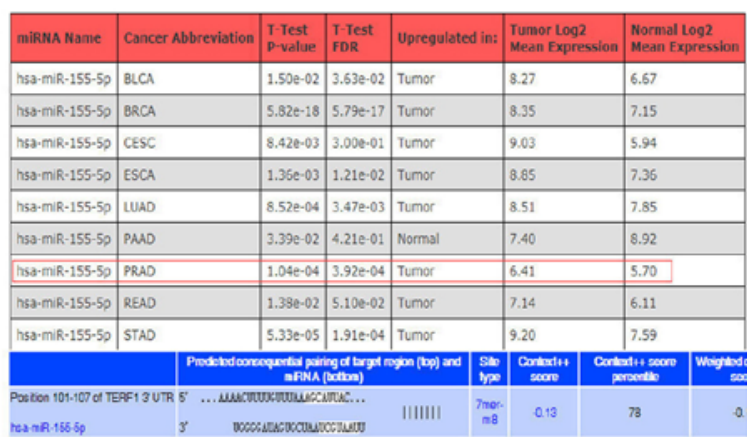

C

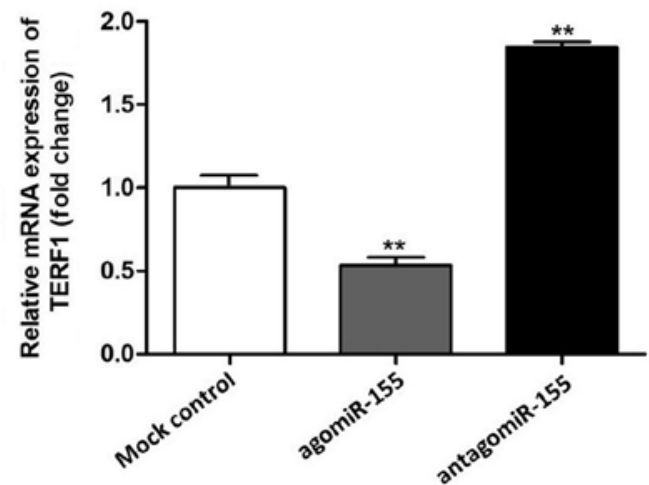

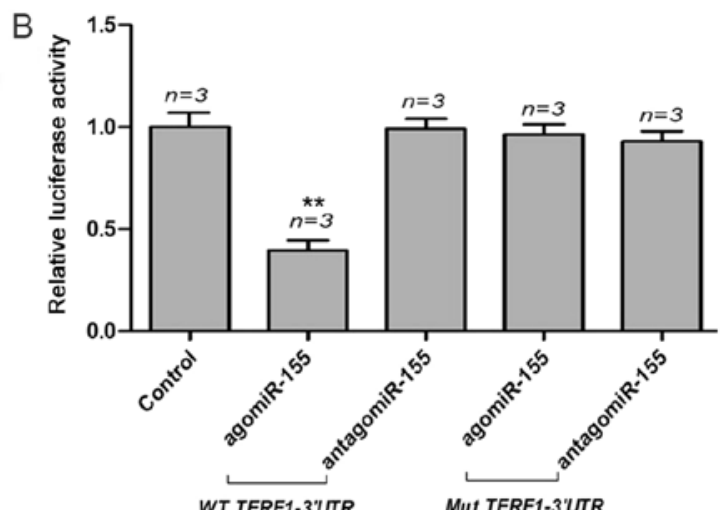

D
Mut TERF1-3'UTR

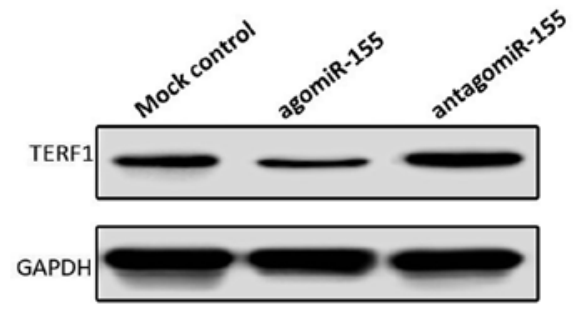

Figure 4. TERF1 3'-UTR is a direct target of miR-155. (A) Bioinformatics prediction and screening of potential miRNAs that target TERF1 by miRWalk and Targetscan databases. (B) Relative luciferase activity of WT-TERF1 3'-UTR and Mut-TERF1 3'-UTR constructs in PC3 cells following the transfection with agomiR-155 or antagomiR-155. ${ }^{* *} \mathrm{P}<0.01$ vs. control. TERF1 (C) mRNA and (D) protein expression levels in PC3 cells were analyzed using reverse transcription-quantitative PCR or western blotting, respectively, following the transfection with agomiR-155 or antagomiR-155. ${ }^{* * *} \mathrm{P}<0.01 \mathrm{vs}$. mock control. TERF1, telomeric repeat binding factor 1; miRNA/miR, microRNA; 3'-UTR, 3'-untranslated region; WT, wild-type; Mut, mutant. 
A
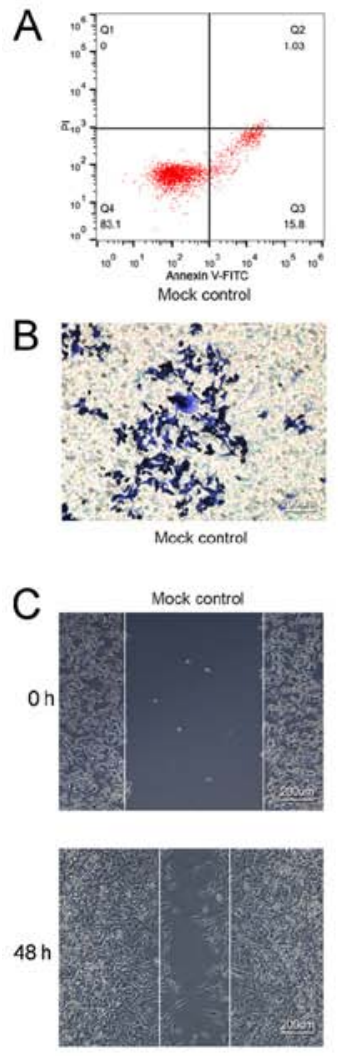

D
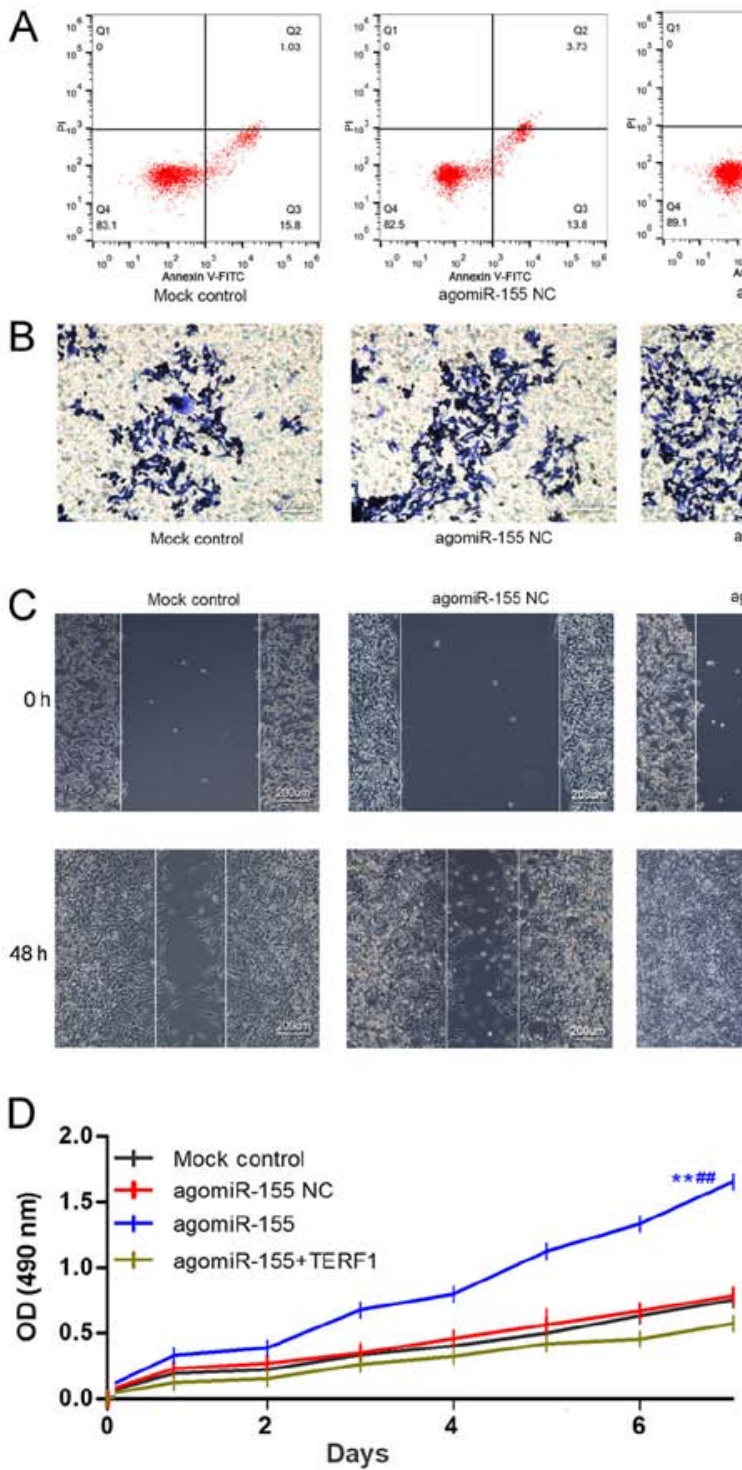

agomiR-155 NC
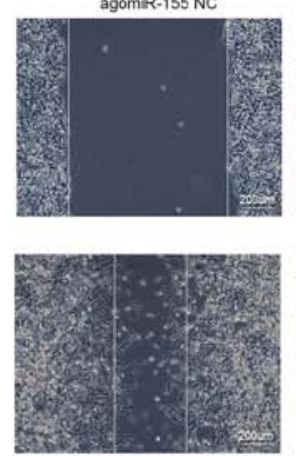

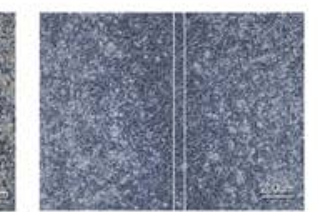

$\mathrm{E}$

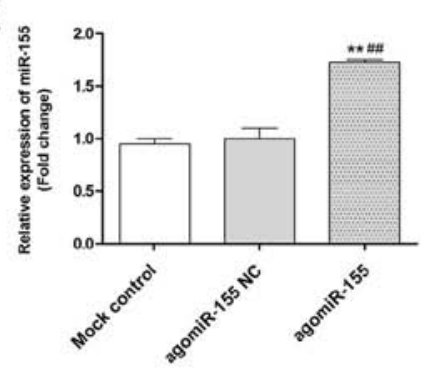

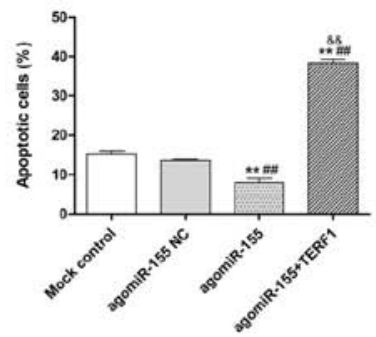
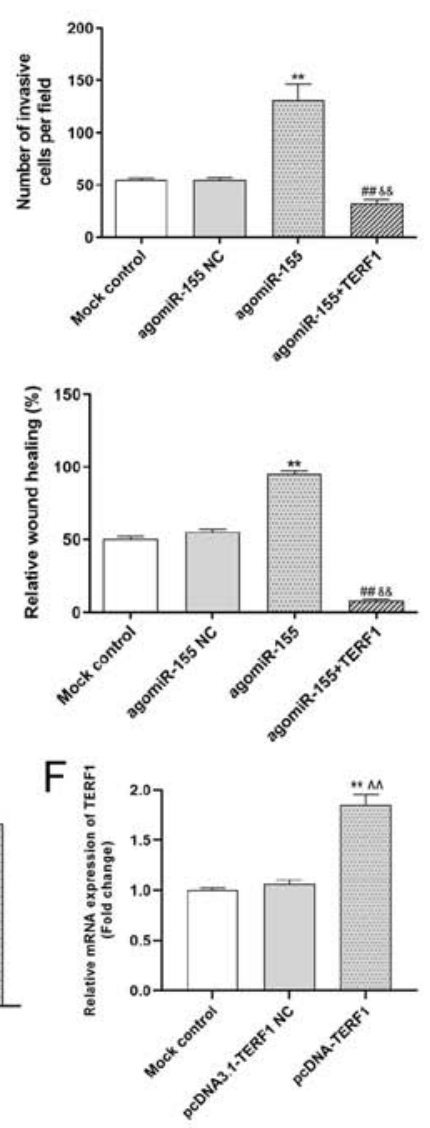

Figure 5. Overexpression of miR-155 promotes the viability, migration and invasion of the PC3 cell line, while inhibiting apoptosis, by targeting TERF1. (A) Levels of apoptosis in PC3 cells in each group were analyzed by flow cytometry. (B) Invasive ability of PC3 cells in each group was analyzed using a Transwell assay. Scale bar, 200- $\mu \mathrm{m}$. (C) Migratory ability of PC3 cells in each group was analyzed using a wound healing assay. Scale bar, 200- $\mu \mathrm{m}$. (D) Viability of PC3 cells in each group was analyzed using an MTT assay. (E) Transfection efficiency of the agomiR-155 in PC3 cells was analyzed using RT-qPCR. (F) Transfection efficiency of pcDNA3.1-TERF1 in PC3 cells was analyzed using RT-qPCR. ${ }^{* *} \mathrm{P}<0.01$ vs. mock control; ${ }^{*} \mathrm{P}<0.01 \mathrm{vs.} \mathrm{agomiR-155} \mathrm{NC;}$ ${ }^{\wedge} \mathrm{P}<0.01$ vs. pcDNA3.1-TERF1 NC. \&\&P<0.01 agomiR-155 vs. agomiR-155+TERF1. TERF1, telomeric repeat binding factor 1; miR, microRNA; RT-qPCR, reverse transcription-quantitative PCR; PI, propidium iodide; NC, negative control; OD, optical density.

\section{Discussion}

The main causes of PCa-associated mortality and poor prognosis in patients are castration-resistant and metastatic PCa $(1,3)$. The median survival time is no more than 2 years for patients with CRPC (22). Therefore, it is necessary to investigate the potential mechanisms of PCa progression and to determine novel biomarkers to specifically identify cases of aggressive $\mathrm{PCa}$.

TERF1 encodes a ubiquitously expressed protein of 439 amino acids that is primarily located at chromosome ends, where it contributes to the protection and maintenance of telomeric DNA $(23,24)$. Several previous studies have reported that the expression levels of TERF1 were often downregulated during the progression of glioblastoma and seminoma (25-27). These results indicated that TERF1 disruption in cancer may be a general phenomenon. Nevertheless, the molecular mechanism remains unclear. Hanahan and Weinberg (8) revealed that the maintenance of telomeres above a minimum length is critical to maintaining the unlimited proliferative potential of cancer cells, and telomeres are therefore considered as potential anticancer targets. In total, $>90 \%$ of human cancers have been discovered to abnormally express telomerase (28), while tumors that do not express telomerase are based on recombination between telomere sequences and activating another extension (29). The results of the present study suggested that TERF1 may serve as a potential tumor suppressor gene in the development of $\mathrm{PCa}$, as significantly downregulated expression levels of TERF1 were identified in PCa tissue compared with benign prostate tissue. The downregulation of TERF1 using siRNA in the current study was further revealed to promote the viability, invasion and migration, while inhibiting 
A

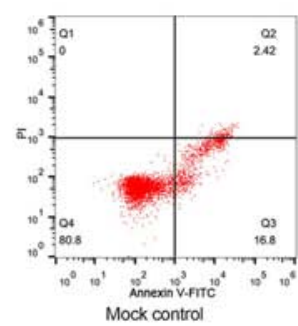

B

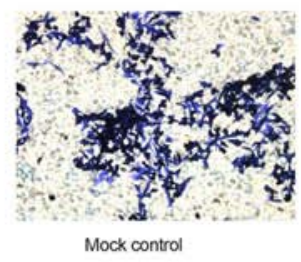

C
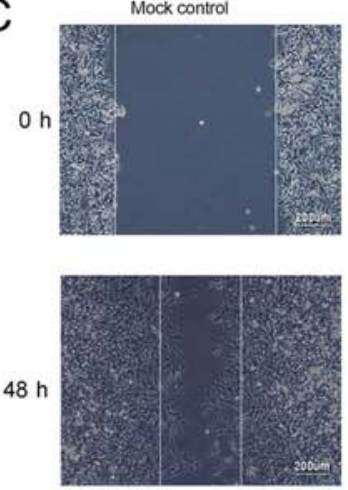

D $\left.{ }^{1.0}\right]+$ Mock control

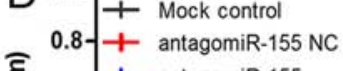

E $\begin{aligned} & 0.8-\text { + } \\ & 0.6-\text { antagomiR- } 155 \mathrm{NC}\end{aligned}$

号

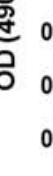

0.4
0.2
0.0

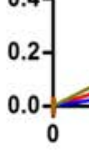

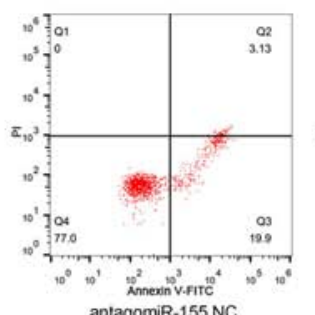

antagomiR-155 NC

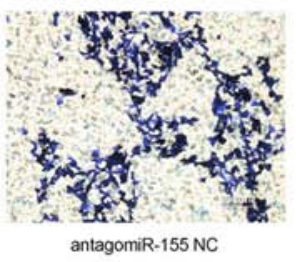

antagomiR-155 NC
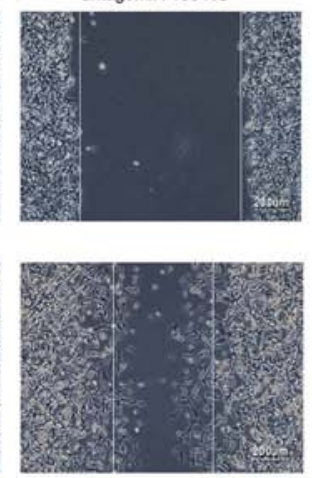
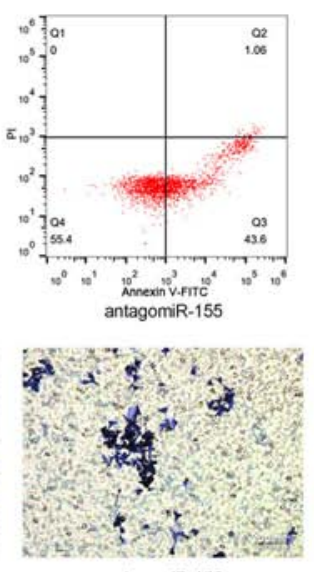

antagomiR-155
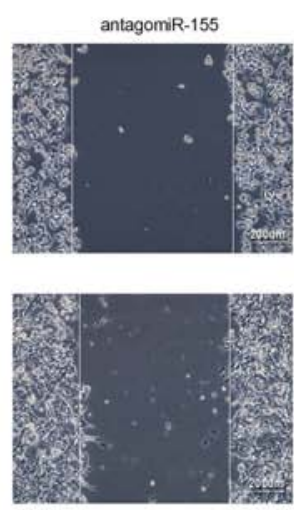
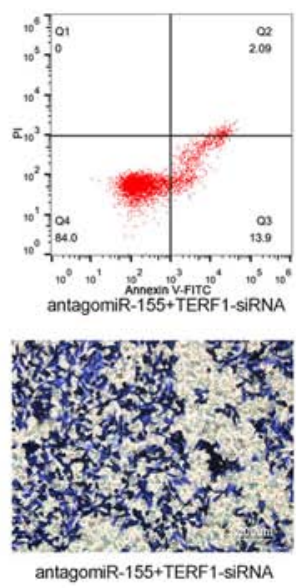

antagomiR-155+TERF1-siRNA
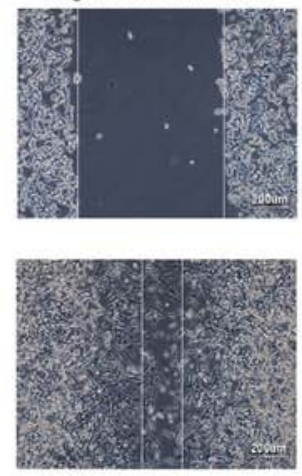

E
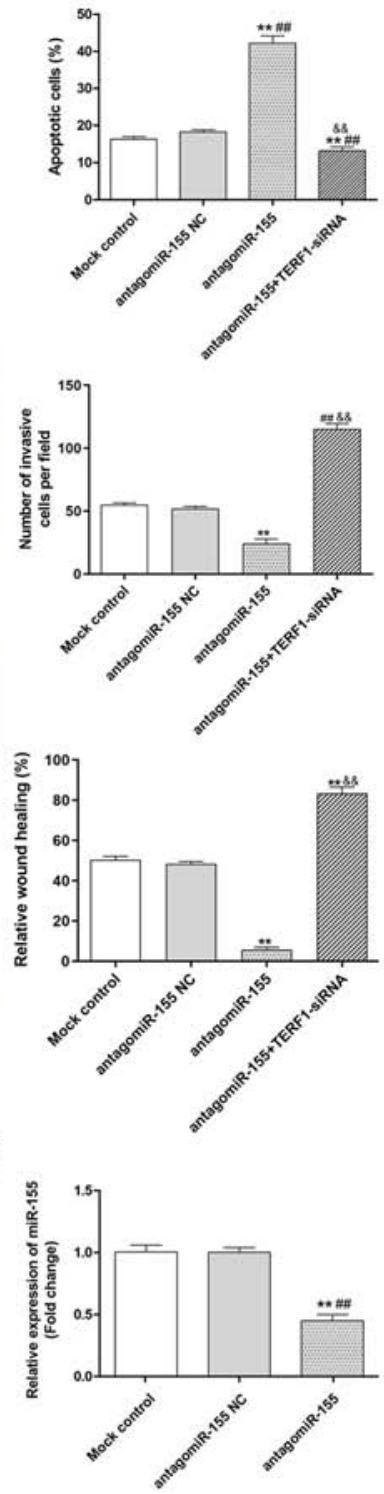

Figure 6. Knockdown of miR-155 inhibits the viability, migration and invasion of the PC3 cell line, while promoting apoptosis, by targeting TERF1. (A) Levels of apoptosis in PC3 cells in each group were analyzed by flow cytometry. (B) Invasive ability of PC3 cells in each group was analyzed using a Transwell assay. Scale bar, 200- $\mu \mathrm{m}$. (C) Migratory ability of PC3 cells in each group was analyzed using a wound healing assay. Scale bar, 200- $\mu \mathrm{m}$. (D) Viability of PC3 cells in each group was analyzed using an MTT assay. (E) Transfection efficiency of antagomiR-155 in PC3 cells was analyzed using RT-qPCR. ${ }^{* *} \mathrm{P}<0.01$ vs. mock control; ${ }^{\#} \mathrm{P}<0.01$ vs. antagomiR-155 NC; ${ }^{\&} \mathrm{P}<0.01$ antagomiR-155 vs. antagomiR-155+TERF1-siRNA.TERF1, telomeric repeat binding factor 1; miR, microRNA; RT-qPCR, reverse transcription-quantitative PCR; PI, propidium iodide; NC, negative control; siRNA, small interfering RNA; OD, optical density.

the apoptosis, of PCa cells. Furthermore, TERF1 knockdown downregulated the expression levels of E-cadherin and upregulated the expression levels of $\mathrm{N}$-cadherin and vimentin, which suggested that the downregulation of TERF1 may promote the progression of $\mathrm{PCa}$ predominantly through the EMT pathway. To further analyze the molecular mechanism of $\mathrm{PCa}$ progression, the interaction between TERF1 and miRNAs was investigated.

miRNAs have been demonstrated to serve an important role in PCa progression. For example, miR-338-3p downregulation promoted the proliferation and invasion of PCa cells (30); and the overexpression of miR-34a inhibited the proliferation, migration and invasion of PCa cells $(31,32)$. miR-765 was reported to be an important mediator for inhibiting growth, migration and invasion in PCa (33). Dinami et al (17) revealed that miR-155 upregulation antagonized telomere integrity by targeting TERF1 in breast cancer. In addition, other previous studies have indicated that miR-155 may serve an oncogenic role in several types of hematological malignancy and solid tumor. The overexpression of miR-155 was previously demonstrated to be associated with cell proliferation, cell invasion, cell death and cell survival (34-36). Furthermore, miR-155 directly targeted and inhibited a series of genes (e.g. FOXO3, SMARCA4 or Ubiquilin-1) to participate in the biological process of tumor development (37-40). To the best of our knowledge, the present study was the first to indicate that TERF1 may be a direct target of miR-155, as confirmed by a dual luciferase reporter assay. Furthermore, a negative association was observed between the expression levels of miR-155 and TERF1. The overexpression of miR-155 was discovered to 
promote PC3 cell viability, invasion and migration, while inhibiting apoptosis. Conversely, the knockdown of miR-155 yielded the opposite results. Therefore, miR-155 was hypothesized to be an oncogene in PCa. The results also revealed that TERF1 could reverse the regulatory effect of miR-155 on the apoptosis, viability, migration and invasion of PC3 cells. Collectively, the findings suggested that miR-155 may exert a carcinogenic role in PCa by targeting and downregulating TERF1.

The local hypoxic environment is important during the progression of $\mathrm{PCa}$. A number of signaling pathways participated in the progression of prostate cancer mediated by miR-155. Hypoxia inducible factor $1 \alpha$ (HIF-1 $\alpha)$ induces the expression of VEGF, which has been reported to promote neovascularization in $\mathrm{PCa}$ (41). This pathological process has been suggested to promote the proliferation and metastasis of PCa targeted by miRNAs (42). Previous studies have illustrated that the overexpression of HIF-1 $\alpha$ increased the risk of CRPC and the metastasis of PCa. In addition, it was also suggested that HIF-1 $\alpha$ may be a potential molecular target of PCa (43). The Notch signaling pathway was discovered to serve an important role in the PCa cell proliferation and apoptosis (44). Nevertheless, there are few studies focusing on the role of the Notch signaling pathway on the invasion and metastasis of PCa. Previous findings have highlighted the involvement of Notch signaling in prostate development and in the maintenance of prostate homeostasis (45). Guo et al (46) reported contradictory roles of Notch signaling, where it served as a tumor-promoting role in human acute lymphoblastic leukaemia or tumor-suppressive role in basal cell carcinoma. Furthermore, studies have reported synergistic positive results in PCa cells when Notch signaling inhibitors were combined with androgen deprivation therapy $(47,48)$.

However, the present study only used one cell line to investigate the potential mechanisms of TERF1 in PCa, which is a limitation of the study. In order to validate the results, both androgen-sensitive and androgen-resistant PCa cell lines should be used in further investigations.

In conclusion, the results of the present study suggested that TERF1 may function as a tumor suppressor in PCa, which suppresses the migration and invasion of PC3 cells through the EMT pathway. Furthermore, miR-155 was discovered to serve an important role in the progression of $\mathrm{PCa}$ via negatively regulating TERF1. Therefore, TERF1 and miR-155 may serve as potential diagnostic biomarkers and prognostic markers in $\mathrm{PCa}$.

\section{Acknowledgements}

The authors would like to thank Professor Wanlong Tan and Professor Fei Li (Nanfang Hospital, Southern Medical University) for their help in guiding experiments and quality control strategies.

\section{Funding}

The present study was supported by the Sichuan Science and Technology Program (grant no. 2018JY0670), the Key Project of Zigong Science and Technology (grant nos. 2016SF07 and 2018SHFZ06), the Scientific Research Subject of Sichuan Medical Association (grant no. S16087), the Science Foundation of Health Commission of Sichuan Province (grant no. 18PJ459) and the Science Foundation of Health Commission of Zigong (grant nos. 2018WJWZD02 and 2018WJWZC08).

\section{Availability of data and materials}

The datasets used and/or analyzed during the current study are available from the corresponding author on reasonable request.

\section{Authors' contributions}

WC and LNH conceptualized the study, performed the experiments and wrote the manuscript. YL and XZ contributed significantly to data analysis and manuscript preparation. CPW, MQS and JHL analyzed the data. YC analyzed data, and prepared the figures and tables.. All authors read and approved the final manuscript.

\section{Ethics approval and consent to participate}

Not applicable.

\section{Patient consent for publication}

Not applicable.

\section{Competing interests}

The authors declare that they have no competing interests.

\section{References}

1. Subudhi SK: New approaches to immunotherapy for metastatic castration-resistant prostate cancer. Clin Adv Hematol Oncol 17: 283-286, 2019.

2. Siegel RL, Miller KD and Jemal A: Cancer statistics, 2018. CA Cancer J Clin 68: 7-30, 2018.

3. He BM, Chen R, Sun TQ, Yang Y, Zhang CL, Ren SC, Gao X and Sun YH: Prostate cancer risk prediction models in Eastern Asian populations: Current status, racial difference, and future directions. Asian J Androl 22: 158-161, 2020.

4. Koo K and Hyams ES: Assessment of men's risk thresholds to proceed with prostate biopsy for the early detection of prostate cancer. Int Urol Nephrol 51: 1297-1302, 2019.

5. Dalla Via J, Daly RM, Owen PJ, Mundell NL, Rantalainen T and Fraser SF: Bone mineral density, structure, distribution and strength in men with prostate cancer treated with androgen deprivation therapy. Bone 127: 367-375, 2019.

6. Grun LK, Teixeira NDR Jr, Mengden LV, de Bastiani MA, Parisi MM, Bortolin R, Lavandoski P, Pierdoná V, Alves LB, Moreira JCF, et al: TRF1 as a major contributor for telomeres' shortening in the context of obesity. Free Radic Biol Med 129: 286-295, 2018.

7. Hu J, Sun L, Wang LM and Jiang SJ: Analysis of the nuclear localization signal of TRF1 in non-small cell lung cancer. Biol Res 42: 217-222, 2009.

8. Hanahan D and Weinberg RA: Hallmarks of cancer: The next generation. Cell 144: 646-674, 2011.

9. Takahashi RU, Prieto-Vila M, Kohama I and Ochiya T: Development of miRNA-based therapeutic approaches for cancer patients. Cancer Sci 110: 1140-1147, 2019.

10. Liu G and Li B: Role of miRNA in transformation from normal tissue to colorectal adenoma and cancer. J Cancer Res Ther 15: 278-285, 2019.

11. Vos PD, Leedman PJ, Filipovska A and Rackham O: Modulation of miRNA function by natural and synthetic RNA-binding proteins in cancer. Cell Mol Life Sci 76: 3745-3752, 2019.

12. Kogure A, Kosaka N and Ochiya T: Cross-talk between cancer cells and their neighbors via miRNA in extracellular vesicles: An emerging player in cancer metastasis. J Biomed Sci 26: 7, 2019. 
13. Wang M, Jiang S, Zhou L, Yu F, Ding H, Li P, Zhou M and Wang K: Potential Mechanisms of Action of Curcumin for Cancer Prevention: Focus on Cellular Signaling Pathways and miRNAs. Int J Biol Sci 15: 1200-1214, 2019.

14. Yu Z, Wang Z, Li F, Yang J and Tang L: miR 138 modulates prostate cancer cell invasion and migration via $\mathrm{Wnt} / \beta$ catenin pathway. Mol Med Rep 17: 3140-3145, 2018.

15. Li L, Tang P, Li S, Qin X, Yang H, Wu C and Liu Y: Notch signaling pathway networks in cancer metastasis: A new target for cancer therapy. Med Oncol 34: 180, 2017.

16. Chen CH, Li SX, Xiang LX, Mu HQ, Wang SB and Yu KY: HIF-1 $\alpha$ induces immune escape of prostate cancer by regulating NCR1/NKp46 signaling through miR-224. Biochem Biophys Res Commun 503: 228-234, 2018.

17. Dinami R, Ercolani C, Petti E, Piazza S, Ciani Y, Sestito R, Sacconi A, Biagioni F, le Sage C, Agami R, et al: miR-155 drives telomere fragility in human breast cancer by targeting TRF1. Cancer Res 74: 4145-4156, 2014

18. Livak KJ and Schmittgen TD: Analysis of relative gene expression data using real-time quantitative PCR and the 2(-Delta Delta C(T)) Method. Methods 25: 402-408, 2001.

19. Dweep H, Sticht C, Pandey P and Gretz N: miRWalk - database: Prediction of possible miRNA binding sites by 'walking' the genes of three genomes. J Biomed Inform 44: 839-847, 2011

20. Chandrashekar DS, Bashel B, Balasubramanya SAH, Creighton CJ, Ponce-Rodriguez I, Chakravarthi BVSK and Varambally S: UALCAN: A Portal for Facilitating Tumor Subgroup Gene Expression and Survival Analyses. Neoplasia 19: 649-658, 2017.

21. Epstein JI, Egevad L, Amin MB, Delahunt B, Srigley JR and Humphrey PA; Grading Committee: The 2014 International Society of Urological Pathology (ISUP) Consensus Conference on Gleason Grading of Prostatic Carcinoma: Definition of Grading Patterns and Proposal for a New Grading System. Am J Surg Pathol 40: 244-252, 2016.

22. Blanker $\mathrm{MH}$ and Bangma $\mathrm{CH}$ : Presence of prostate cancer, but absence of active treatment. Ned Tijdschr Geneeskd 163: D3698, 2019 (In Dutch).

23. Wang L, Tu Z, Liu C, Liu H, Kaldis P, Chen Z and Li W: Dual roles of TRF1 in tethering telomeres to the nuclear envelope and protecting them from fusion during meiosis. Cell Death Differ 25: 1174-1188, 2018.

24. Lee YW, Arora R, Wischnewski H and Azzalin CM: TRF1 participates in chromosome end protection by averting TRF2-dependent telomeric R loops. Nat Struct Mol Biol 25: 147-153, 2018.

25. Bejarano L, Schuhmacher AJ, Méndez M, Megías D, Blanco-Aparicio C, Martínez S, Pastor J, Squatrito M and Blasco MA: Inhibition of TRF1 Telomere Protein Impairs Tumor Initiation and Progression in Glioblastoma Mouse Models and Patient-Derived Xenografts. Cancer Cell 32: 590-607.e4, 2017.

26. Liu Q, Wang G, Lyu Y, Bai M, Jiapaer Z, Jia W, Han T, Weng R, Yang Y, Yu Y, et al: The miR-590/Acvr2a/Terf1 Axis Regulates Telomere Elongation and Pluripotency of Mouse iPSCs. Stem Cell Reports 11: 88-101, 2018.

27. Chan FL, Vinod B, Novy K, Schittenhelm RB, Huang C, Udugama M, Nunez-Iglesias J, Lin JI, Hii L, Chan J, et al: Aurora Kinase B, a novel regulator of TERF1 binding and telomeric integrity. Nucleic Acids Res 45: 12340-12353, 2017.

28. Kim NW, Piatyszek MA, Prowse KR, Harley CB, West MD Ho PL, Coviello GM, Wright WE, Weinrich SL and Shay JW: Specific association of human telomerase activity with immortal cells and cancer. Science 266: 2011-2015, 1994.

29. Bryan TM, Englezou A, Dalla-Pozza L, Dunham MA and Reddel RR: Evidence for an alternative mechanism for maintaining telomere length in human tumors and tumor-derived cell lines. Nat Med 3: 1271-1274, 1997.

30. Cai C, Zhi Y, Wang K, Zhang P, Ji Z, Xie C and Sun F: CircHIPK3 overexpression accelerates the proliferation and invasion of prostate cancer cells through regulating miRNA-338-3p. OncoTargets Ther 12: 3363-3372, 2019.

31. Zhu M, Zheng Z, Huang J, Ma X, Huang C, Wu R, Li X, Liang Z, Deng F, Wu J, et al: Modulation of miR-34a in curcumin-induced antiproliferation of prostate cancer cells. J Cell Biochem 120: 15616-15624, 2019.
32. Li N, Zhang LY, Qiao YH and Song RJ: Long noncoding RNA LINC00662 functions as miRNA sponge to promote the prostate cancer tumorigenesis through targeting miR-34a. Eur Rev Med Pharmacol Sci 23: 3688-3698, 2019.

33. Leung YK, Chan QK, Ng CF, Ma FM, Tse HM, To KF, Maranchie J, Ho SM and Lau KM: Correction: Hsa-miRNA-765 as a Key Mediator for Inhibiting Growth, Migration and Invasion in Fulvestrant-Treated Prostate Cancer. PLoS One 14: e0214184, 2019.

34. Witten LW, Cheng CJ and Slack FJ: miR-155 drives oncogenesis by promoting and cooperating with mutations in the c-Kit oncogene. Oncogene 38: 2151-2161, 2019.

35. Witten L and Slack FJ: miR-155 as a novel clinical target for hematological malignancies. Carcinogenesis 41: 2-7, 2020.

36. Jurkovicova D, Magyerkova M, Kulcsar L, Krivjanska M, Krivjansky V, Gibadulinova A, Oveckova I and Chovanec M: miR-155 as a diagnostic and prognostic marker in hematological and solid malignancies. Neoplasma 61: 241-251, 2014.

37. Zhang Y, Zhao $\mathrm{H}$ and Zhang L: Identification of the tumor suppressive function of circular RNA FOXO3 in non small cell lung cancer through sponging miR-155. Mol Med Rep 17: 7692-7700, 2018

38. Yang YP, Nguyen PNN, Ma HI, Ho WJ, Chen YW, Chien Y, Yarmishyn AA, Huang PI, Lo WL, Wang CY, et al: Tumor Mesenchymal Stromal Cells Regulate Cell Migration of Atypical Teratoid Rhabdoid Tumor through Exosome-Mediated miR155/SMARCA4 Pathway. Cancers (Basel) 11: E720, 2019.

39. Yadav S, Singh N, Shah PP, Rowbotham DA, Malik D, Srivastav A, Shankar J, Lam WL, Lockwood WW and Beverly LJ: MIR155 Regulation of Ubiquilin1 and Ubiquilin2: Implications in Cellular Protection and Tumorigenesis. Neoplasia 19: 321-332, 2017.

40. Wang Y, Yan L, Zhang L, Xu H, Chen T, Li Y, Wang H, Chen S, Wang W, Chen C, et al: NT21MP negatively regulates paclitaxel-resistant cells by targeting miR $1553 \mathrm{p}$ and $\mathrm{miR}$ $155-5 p$ via the CXCR4 pathway in breast cancer. Int J Oncol 53: 1043-1054, 2018.

41. Deep G, Kumar R, Nambiar DK, Jain AK, Ramteke AM, Serkova NJ, Agarwal C and Agarwal R: Silibinin inhibits hypoxia-induced HIF- $1 \alpha$-mediated signaling, angiogenesis and lipogenesis in prostate cancer cells: In vitro evidence and in vivo functional imaging and metabolomics. Mol Carcinog 56: 833-848, 2017

42. Hasan D, Gamen E, Abu Tarboush N, Ismail Y, Pak O and Azab B: PKM2 and HIF-1 $\alpha$ regulation in prostate cancer cell lines. PLoS One 13: e0203745, 2018

43. Chen B, Zhang M, Xing D and Feng Y: Atorvastatin enhances radiosensitivity in hypoxia-induced prostate cancer cells related with HIF-1 $\alpha$ inhibition. Biosci Rep 37: BSR20170340, 2017.

44. Mohamed AA, Tan SH, Xavier CP, Katta S, Huang W, Ravindranath L, Jamal M, Li H, Srivastava M, Srivatsan ES, et al: Synergistic activity with NOTCH inhibition and androgen ablation in ERG-positive prostate cancer cells. Mol Cancer Res 15: 1308-1317, 2017.

45. Deng G, Ma L, Meng Q, Ju X, Jiang K, Jiang P and Yu Z: Notch signaling in the prostate: Critical roles during development and in the hallmarks of prostate cancer biology. J Cancer Res Clin Oncol 142: 531-547, 2016.

46. Guo H, Lu Y, Wang J, Liu X, Keller ET, Liu Q, Zhou Q and Zhang J: Targeting the Notch signaling pathway in cancer therapeutics. Thorac Cancer 5: 473-486, 2014.

47. Kita Y, Goto T, Akamatsu S, Yamasaki T, Inoue T, Ogawa O and Kobayashi T: Castration-resistant prostate cancer refractory to second-generation androgen receptor axis-targeted agents: Opportunities and challenges. Cancers (Basel) 10: 345, 2018

48. Domingo-Domenech J, Vidal SJ, Rodriguez-Bravo V, Castillo-Martin M, Quinn SA, Rodriguez-Barrueco R, Bonal $\mathrm{DM}$, Charytonowicz E, Gladoun N, de la Iglesia-Vicente J, et al: Suppression of acquired docetaxel resistance in prostate cancer through depletion of notch- and hedgehog-dependent tumor-initiating cells. Cancer Cell 22: 373-388, 2012.

This work is licensed under a Creative Commons Attribution-NonCommercial-NoDerivatives 4.0 International (CC BY-NC-ND 4.0) License. 